\title{
Cirugía ambulatoria: ¿Qué hacer en el período posoperatorio? Desde el término de la cirugía hasta el control domiciliario
}

\author{
Alejandro González A., Maximiliano Zamora H., \\ José Ignacio Contreras C. y Mauricio Ibacache F.
}

Departamento de Anestesiología. Facultad de Medicina. Pontificia Universidad Católica de Chile. Santiago, Chile.

Recibido el 12 de junio de 2017, aceptado para publicación el 4 de diciembre de 2017

Correspondencia a: Dr. Alejandro González A. gonzalea@med.puc.c

Outpatient surgery: What to do in the postoperative period? From the end of surgery to home surveillance

Postoperative recovery and post discharge surveillance and support are essential in outpatient surgery success. The postoperative recovery process is divided into Phase I and Phase II, in which predefined clinical criteria are used to decide whether a patient can be moved to the next phase or be discharged to home, respectively. At discharge, postoperative indications are given in writing and an adult must accompany the patient. After discharge, the patient is monitored during the first few days, usually through a telephone call and predefined clinical parameters are evaluated. Outpatient surgery outcome evaluation is performed through specific indicators such as prolonged postoperative stay, unanticipated hospital admission, and return to hospital and readmission.

Key words: outpatient surgery; postoperative recovery; postoperative discharge; post discharge followup; adverse outcomes.

\section{Resumen}

La recuperación posoperatoria, el control y apoyo posalta son esenciales en el éxito de la cirugía ambulatoria. El proceso de recuperación posoperatoria se divide en recuperación Fase I y Fase II, en las cuales se utilizan criterios clínicos predefinidos para decidir si un paciente puede ser trasladado a la fase siguiente o ser dado de alta al domicilio, respectivamente. Al momento del alta, las indicaciones posoperatorias se entregan por escrito y el paciente debe estar acompañado por un adulto. Después del alta, se realiza un seguimiento del paciente durante los primeros días, habitualmente a través de llamado telefónico, evaluando parámetros clínicos predefinidos. La evaluación de resultados en cirugía ambulatoria se realiza a través de indicadores específicos, tales como retardo del alta, hospitalización no programada y rehospitalización posalta.

Palabras clave: cirugía ambulatoria; recuperación posoperatoria; alta posoperatoria; seguimiento posalta; eventos adversos.

\section{Introducción}

El progreso de las técnicas quirúrgicas, de los fármacos y técnicas anestésicas $\mathrm{y}$, de manera importante, los cuidados perioperatorios han contribuido al desarrollo y expansión de la cirugía ambulatoria (CA) a nivel mundial ${ }^{1,2}$. Entendida como aquellos procedimientos quirúrgicos en los que la admisión, la cirugía y el alta del paciente ocurren el mismo día, la CA representa más de los $60 \%$ del total de cirugías electivas en países desarrollados ${ }^{3}$. Si bien, no contamos con estadísticas nacionales al respecto, creemos que en nuestro país existe un creciente interés por realizar $\mathrm{CA}^{4,5}$.

El proceso de la CA, incluida la anestesia y la cirugía propiamente tal, ha demostrado ser seguro, eficiente y rentable ${ }^{2}$. Comparada con la cirugía intrahospitalaria, tiene ventajas adicionales, para pacientes e instituciones de salud, tales como: un menor costo económico, recuperación más rápida 
del estado fisiológico previo, menor morbimortalidad, mayor satisfacción usuaria, liberación de camas de hospitalización, entre otras ${ }^{6}$. Lograr estos beneficios requiere de una cuidadosa selección de pacientes y cirugías, contar con una infraestructura y equipamiento con los mismos estándares de seguridad y calidad de los procedimientos quirúrgicos de pacientes hospitalizados ${ }^{1,7,8}$.

Consideramos que la CA se soporta en tres principios fundamentales, que son: 1) la calidad del centro o servicio donde se realiza el procedimiento quirúrgico; 2) la adecuada y meticulosa selección de pacientes y cirugías a realizar, y 3 ) el sistema de control y apoyo posterior al alta. Los dos primeros ítems han sido revisados en números anteriores de esta revista ${ }^{7,8}$. En este artículo revisaremos las etapas de recuperación posoperatoria, los requisitos para el traslado y el alta del paciente, el apoyo y control posterior que requiere un paciente sometido a $\mathrm{CA}$. Además, revisaremos los indicadores que se utilizan para evaluar los resultados de la CA.

\section{Recuperación posoperatoria y alta}

La recuperación posoperatoria es un proceso continuo que comienza con el término de la cirugía y finaliza cuando el paciente retorna a su estado fisiológico preoperatorio. En CA este proceso se divide en dos etapas consecutivas denominadas recuperación Fase I y recuperación Fase II.

\section{Recuperación Fase I o precoz}

Esta fase ocurre en la sala de recuperación posoperatoria habitual, requiere monitorización continua de signos vitales, supervisión médica y de enfermería. Comienza con la llegada del paciente desde pabellón y finaliza cuando el paciente está en condiciones de ser trasladado a una unidad de menor complejidad. Este período permite que el paciente despierte de la anestesia, recupere los reflejos protectores de la vía aérea, reasuma actividad motora, se maneje el dolor y las náuseas y vómitos posoperatorios (NVPO). Para evaluar si un paciente se puede trasladar a una unidad de menor complejidad, se utilizan criterios clínicos predefinidos, organizados en sistemas de puntuación o escalas reproducibles y validadas para su uso clínico. La más conocida $\mathrm{y}$, probablemente, más utilizada es la Escala Modificada de Aldrete ${ }^{9,10}$ que considera el estado de conciencia, el nivel de actividad, parámetros circulatorios, características de la respiración y los niveles de oxigenación. Por otra parte, White et al., desarrollaron una escala de recuperación abreviada ("fast track") con 7 criterios, que añade la intensidad del dolor y la presencia de náuseas y vómitos posoperatorios (NVPO) a los parámetros mencionados ${ }^{9,11}$. White et al otorgan 0 a 2 puntos a cada parámetro, siendo 14 puntos el máximo de la escala. Se requiere un puntaje igual o mayor a 12 y ningún parámetro con puntaje cero para que un paciente sea trasladado a la fase siguiente.

\section{Recuperación Fase II o prealta}

Se inicia con el traslado del paciente desde la sala de recuperación fase I a una unidad de menor complejidad y finaliza cuando el paciente es dado de alta a su domicilio. Esta fase transcurre, generalmente, en una sala equipada con sillones clínicos individuales, oxígeno y aspiración central para cada unidad, monitorización de signos vitales intermitente y supervisión de enfermería. En esta unidad el paciente es realimentado y se prepara para el alta de acuerdo a la evaluación de los siguientes parámetros: estabilidad de sus signos vitales, estado de alerta y deambulación (según tipo de cirugía), estado de la herida operatoria, ausencia de dolor o que pueda ser controlado con analgésicos orales y ausencia de NVPO. Para decidir si un paciente está en condiciones de ser dado de alta se utilizan criterios predefinidos, organizados en escalas de puntuación, similares a los de la fase anterior ${ }^{9,12,13}$. Marshall et $\mathrm{al}^{13}$, desarrollaron una escala que evalúa signos vitales, nivel de actividad, presencia de NVPO, dolor posoperatorio y sangrado de la herida operatoria. A cada parámetro le otorga 0 a 2 puntos, alcanzando un puntaje máximo de 10 puntos. Se requiere un puntaje igual o mayor a 9 puntos para dar de alta a un paciente. En nuestra institución utilizamos la escala de White et al y la de Marshall et al en la recuperación fase I y fase II respectivamente.

Es importante señalar que el trasladado desde recuperación fase I y el alta desde la fase II debe ser evaluado y autorizado por un médico, habitualmente un anestesista. Finalmente, para dar de alta a un paciente son obligatorios los siguientes requisitos ${ }^{14,15}$ :

1. Que un adulto lo acompañe al momento del alta.

2. Estar acompañado de un adulto las primeras $24 \mathrm{~h}$ posoperatorias.

3. Tener un medio de transporte (vehículo) para trasladarse a su domicilio.

4. Tener un domicilio ubicado a 30 a $40 \mathrm{~min}$, en vehículo, del centro de cirugía ambulatorio respectivo o de un servicio de urgencia asociado.

No cumplir con alguno de estos requisitos implica que el paciente se debe quedar hospitalizado la primera noche posoperatoria. 
La recuperación abreviada, vía rápida o "fast track" es una modalidad de recuperación posoperatoria en cirugía ambulatoria, en que el paciente es trasladado directamente desde el pabellón a la recuperación fase II. Las características de la cirugía, los fármacos anestésicos utilizados y la evolución clínica permiten que el paciente alcance criterios de traslado a la fase II en el pabellón, saltándose la etapa de recuperación precoz o fase I. Adicional a la escala de White et al. para recuperación abreviada, Apfelbaum et al ${ }^{16}$ propusieron los siguientes criterios: paciente orientado en tiempo y espacio; dolor ausente o manejable con analgesia oral; ausencia de sangrado activo; signos vitales estables; NVPO leve o ausente; ausencia de relajación muscular residual y saturación de oxígeno mayor o igual a $94 \%$ con aire ambiental o en el nivel preoperatorio. El paciente debe cumplir todos los criterios en pabellón para poder ser trasladado directamente a la recuperación Fase II. Las ventajas de esta modalidad de recuperación son las siguientes: reduce la carga de trabajo del personal; disminuye los costos hospitalarios al paciente y al centro de salud; disminuye la incidencia de hospitalización no programada ${ }^{17,18}$ y es igualmente segura, en términos de morbimortalidad, que la modalidad sin recuperación abreviada ${ }^{16}$.

\section{Indicaciones, apoyo y control posterior al alta}

Al momento del alta, las indicaciones deben ser entregadas en forma verbal y escrita, tanto al paciente como a su acompañante, educando sobre reposo/ actividad, alimentación, manejo del dolor y náuseas posoperatorias, y qué hacer en caso de complicaciones. Además, se le entrega un número de contacto telefónico directo para las primeras $48 \mathrm{~h}$.

El control posterior al alta es una prolongación de la recuperación posoperatoria y es parte fundamental dentro de la CA. Este proceso de seguimiento permite entregar consejos adicionales, tranquiliza al paciente y a su entorno, incrementa la satisfacción del paciente y facilita el mejoramiento de la práctica clínica ${ }^{19}$. Existen varias modalidades descritas de control posalta, que abarcan desde el control telefónico hasta los sistemas de hospitalización domiciliaria.

En nuestra institución, el control posalta es realizado por personal de enfermería mediante un llamado telefónico el día de la cirugía (2-4 h después del alta), el primero y el segundo día posoperatorio. El objetivo de este control es detectar posibles complicaciones, controlar parámetros predefinidos, reforzar o entregar indicaciones según la alteración encon- trada y cerrar el circuito de atención de enfermería. Diseñamos una escala de puntuación utilizando 4 parámetros predefinidos (Tabla 1), que no ha sido validada ni publicada, pero que ha sido muy útil para objetivar, registrar y transmitir la información obtenida en cada control telefónico. Además, se evalúa en forma descriptiva las características de la herida operatoria. Toda la información recopilada en el control telefónico se registra sistemáticamente en un documento ad-hoc, se guarda y se entrega a la persona encargada del siguiente control (Tabla 2).

Recientemente se ha propuesto el uso de aplicaciones para dispositivos móviles para monitorizar de la calidad de recuperación posoperatoria en el domicilio. Los pacientes se autoevalúan diariamente a través de un cuestionario de calidad de recuperación y se fotografían el sitio quirúrgico con la misma periodicidad. El sistema es aplicable y aceptable tanto para pacientes como para el equipo quirúrgico a cargo, con una alta satisfacción de los pacientes y detección adecuada de complicaciones posoperatorias $^{20}$.

\section{Indicadores de resultado en cirugía ambulatoria}

Las complicaciones graves (falla respiratoria, infarto agudo al miocardio, tromboembolismo pulmonar, arritmias severas, etc.) y la mortalidad son muy poco frecuentes en CA. Se reportan incidencias

\section{Tabla 1. Parámetros control posalta}

\begin{tabular}{|lc|}
\hline Parametro & Puntaje \\
Alimentación & \\
Habitual & 3 \\
Blanda, liviana & 2 \\
Solo líquidos & 1 \\
No puede ingerir nada & 0 \\
Actividad & \\
Habitual & 2 \\
Restringida & 1 \\
En reposo & 0 \\
Dolor (EVA*) & \\
Nada & 3 \\
Leve: $<3$ & 2 \\
Moderado: 4-6 & 1 \\
Severo: $>7$ & 0 \\
Náuseas $y$ vómitos & \\
Nada & 3 \\
Mínimo: no requiere tratamiento & 2 \\
Moderado: requiere y responde a tratamiento & \\
oral & 1 \\
Severo: no responde a tratamiento & 0 \\
\hline
\end{tabular}

Si algún parámetro tiene 0 punto, el paciente debe acudir al hospital. *EVA = escala visual análoga. 
Tabla 2. Hoja de registro para control posalta de cirugía ambulatoria

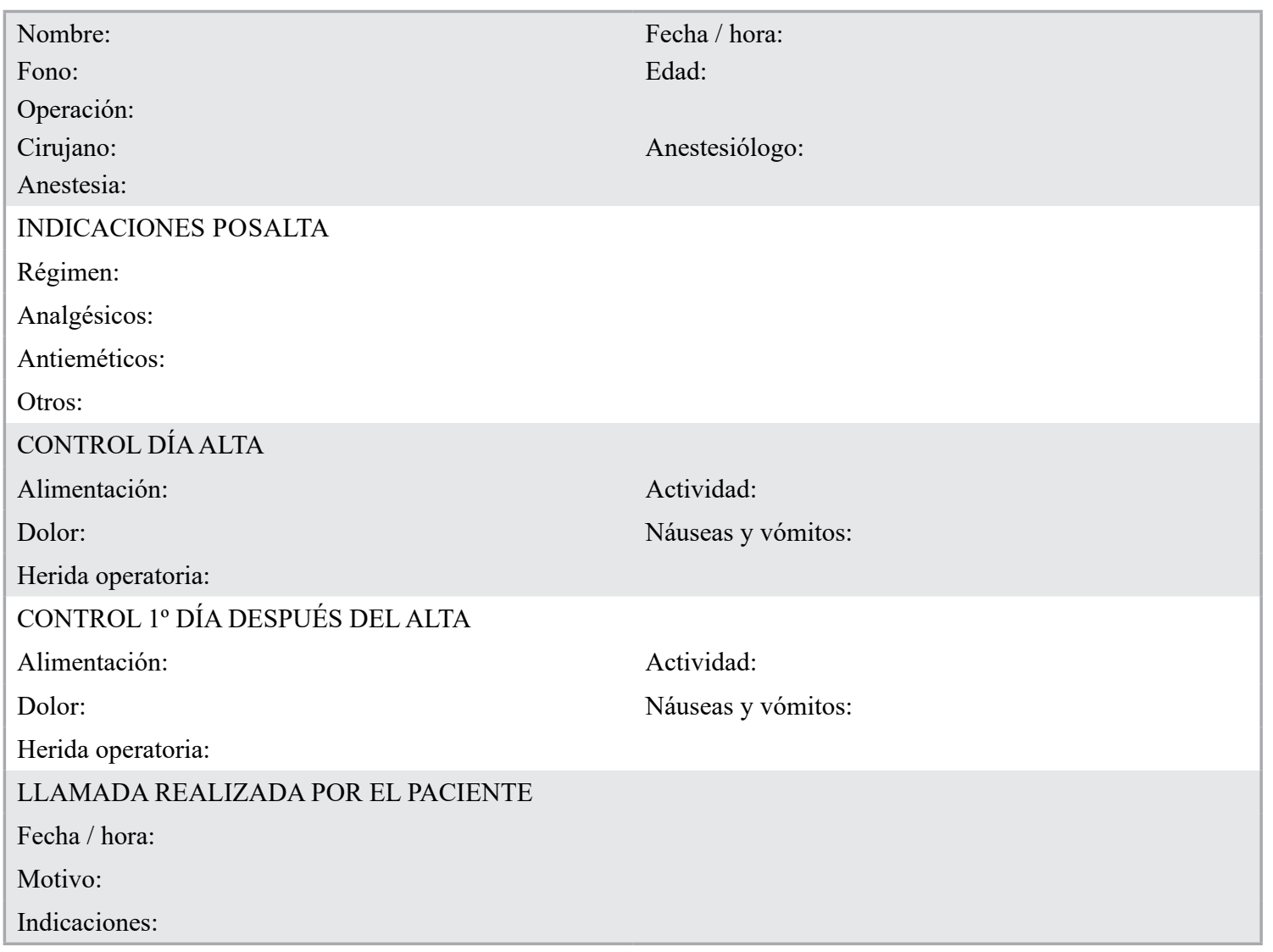

de efectos adversos de $0,08 \%$ y de mortalidad de 1 : 11.273 casos $^{6,21}$. Por esta razón, se utilizan otros indicadores, que son más fidedignos, para evaluar los resultados de la CA tales como el retardo del alta, la hospitalización no programada y la hospitalización posalta ${ }^{6}$.

\section{Retardo del alta}

El 82\% de los pacientes sometidos a CA son dados de alta a su domicilio a las dos horas de finalizada la cirugía y un 95,6\% a las tres horas posterior al término de la operación ${ }^{6}$. El retardo del alta se puede producir por causas preoperatorias, intraoperatorias y posoperatorias. En las de origen preoperatorio destacan las cirugías otorrinolaringológicas, la edad avanzada, el género femenino y pacientes con antecedentes de insuficiencia cardiaca congestiva ${ }^{6,22,23}$. Son causas intraoperatorios la anestesia general, la duración de la cirugía y la anestesia espinal. En los motivos posoperatorios se encuentran el dolor, NVPO, somnolencia y la ausencia de un acompañante adulto al momento del alta ${ }^{6,23,24}$.
El dolor posoperatorio es responsable de retraso en el traslado desde la fase I y del alta desde la fase II en un $30 \%$ y $13 \%$, respectivamente. Los procedimientos quirúrgicos ortopédicos tienen alta incidencia de dolor posoperatorio, particularmente las cirugías de hombro y en aquellas donde se realiza extracción quirúrgica de piezas metálicas ortopédicas. La duración de la CA también está relacionada con la incidencia de dolor posoperatorio; un 10\% de los pacientes cuya cirugía duro más de $90 \mathrm{~min}$ presentan dolor severo ${ }^{25}$.

La incidencia de NVPO en CA varía entre 30$50 \%{ }^{22}$. Apfel et al describieron una puntuación de riesgo para predecir la ocurrencia de NVPO, con los siguientes 4 factores: sexo femenino, historia previa de NVPO, no fumar y uso de opioides en el posoperatorio $^{26}$. El riesgo de desarrollar esta complicación aumenta progresivamente según el número de factores que el paciente presente, desde un $10 \%$ en pacientes con ningún criterio hasta $79 \%$ de riesgo en aquellos que presentan los cuatro factores mencionados. Existen también otros predictores importantes 
de NVPO tales como duración de cirugía mayor de $60 \mathrm{~min}$, cirugía laparoscópica ginecológica, colecistectomía laparoscópica, cirugía intraabdominal y cirugía de oído medio ${ }^{27,28}$.

Es importante reconocer las causales de retraso del alta, prevenirlas y tratarlas oportunamente, de manera de no prolongar la estadía hospitalaria en pacientes sometidos a CA.

\section{Hospitalización no programada}

La hospitalización no programada se refiere a la necesidad de hospitalizar, al menos por una noche, a pacientes sometidos a CA debido a problemas de origen quirúrgico, anestésico, social y/o médico ${ }^{6,29}$. Fortier et $\mathrm{al}^{30}$ reportaron una incidencia de $1,4 \%$ de hospitalización no programada, cuyas causas más frecuentes, en orden decreciente, fueron: dolor posoperatorio, sangrado quirúrgico, NVPO, somnolencia excesiva, complicaciones de enfermedad preexistente y ausencia de acompañante adulto. Los predictores significativos de hospitalización no programada fueron: género masculino; ASA II-III; duración de la anestesia mayor de 1 hora y cirugía que termina después de las $3 \mathrm{pm}$; cirugías otorrinolaringológicas, urológicas, plásticas, traumatológicas y oftalmológicas; sangrado posoperatorio; dolor posoperatorio severo; NVPO y somnolencia excesiva en el posoperatorio.

Una adecuada selección de pacientes, uso de técnicas quirúrgicas mínimamente invasivas y la implementación de un plan de manejo para prevenir y/o tratar posibles complicaciones posoperatorias, pueden reducir la probabilidad de una hospitalización no programada, aumenta la satisfacción por parte de los pacientes y disminuye los costos asociados a la hospitalización.

\section{Hospitalización posterior al alta}

Se entiende por hospitalización posalta a la admisión hospitalaria de pacientes sometidos a CA, debido a complicaciones que ocurren durante los 30 días posteriores a la cirugía ${ }^{6}$. La incidencia de hospitalización posalta reportada por Twersky et $\mathrm{al}^{31}$ fue $1,3 \%$, principalmente debido a complicaciones propias del procedimiento quirúrgico y no reportaron admisiones hospitalarias relacionadas con problemas anestésicos. En otro estudio se señala que los pacientes sometidos a procedimientos urológicos, tales como la resección transuretral de tumores de vejiga y las cistoscopias, tienen una alta probabilidad de readmisión hospitalaria debido a hematuria, retención urinaria e infecciones ${ }^{32}$. Otros autores reportan que la cirugía general, otorrinolaringológica y urológica son las especialidades con la más alta tasa de reingreso hospitalarios post CA con un $3,2 \%, 3,1 \%$ y $2,9 \%$ respectivamente ${ }^{33}$. Por otra, Fleisher et al describieron que los factores asociados con riesgo elevado de hospitalización posalta son la edad avanzada ( $\geq 65$ años), enfermedad vascular periférica, cardiopatía, enfermedad cerebrovascular, patología oncológica y pacientes VIH positivo; la anestesia general también se relacionaría con riesgo aumentado de readmisión hospitalaria ${ }^{34}$.

En resumen, el proceso de recuperación posoperatoria en CA tiene etapas claramente definidas, que requieren criterios clínicos claramente establecidos para transitar de una fase a otra. El alta al domicilio, la red de apoyo familiar, el control y seguimiento posterior debe ser estricto y riguroso, de manera de permitir una recuperación posoperatoria tranquila y segura. La evaluación de resultados en CA se realiza a través de indicadores que evalúan globalmente el proceso quirúrgico, incluyendo la selección de pacientes y cirugías a realizar, el equipamiento e infraestructura utilizados, la selección de las técnicas de anestesia y analgesia, los cuidados posoperatorios y la rigurosidad con que el paciente transita a través de las etapas de la recuperación posoperatoria.

\section{Responsabilidades éticas}

Protección de personas y animales. Los autores declaran que para esta investigación no se han realizado experimentos en seres humanos ni en animales.

Confidencialidad de los datos. Los autores declaran que en este artículo no aparecen datos de pacientes.

Derecho a la privacidad y consentimiento informado. Los autores declaran que en este artículo no aparecen datos de pacientes.

\section{Conflictos de interés}

Los autores declaran no tener conflictos de interés. 


\section{Referencias}

1. De Lathouwer C, Poullier JP. How much ambulatory surgery in the World in 19961997 and trends? Ambulatory Surgery 2000;8:191-210.

2. White PF, Eng MR. Ambulatory (Outpatient) Anesthesia. En: Miller RD, ed. Miller's Anesthesia 7th Ed. Philadelphia: Elseiver Churchill Livingstone, 2010;2419-59.

3. Cullen KA, Hall MJ, Golosinskiy A. Ambulatory surgery in the United States, 2006. Natl Health Stat Reports 2009;11:125.

4. Brunetto B, Castilla A, Sacaluga F. La adenoamigdalectomía como un procedimiento ambulatorio. Rev Otorrinolaringol Cir Cabeza Cuello 1992;52:167-71.

5. Patillo J, Kusanovic R, Salas P, Reyes J, García-Huidobro I, Sanhueza M et al. Colecistectomía laparoscópica ambulatoria: Una experiencia factible en un hospital público chileno. Rev Med Chile 2004;132:429-36.

6. Shnaider I, Chung F. Outcomes in day surgery. Curr Opin Anaesthesiol. 2006; 19:622-9.

7. Nazar C, González A, Zamora M. Cirugía ambulatoria: selección de pacientes y procedimientos quirúrgicos. Rev Chil Cir. 2015;67:207-13.

8. González A, De la Fuente N, Del Río E, Zamora M, Nazar C. Cómo planificar, diseñar y organizar un centro de cirugía ambulatoria. Rev Chil Cir. 2016;68:32832.

9. McGrath B, Chung F. Postoperative recovery and discharge. Anesthesiology Clin N Am. 2003; 21: 367-86.

10. Aldrete JA. The post anaesthesia recovery score revisited. J Clin Anesth. 1995;7:8991.

11. White PF, Song D. New Criteria for fast-tracking after outpatient Anesthesia: a comparison with the Modified Aldrete's Scoring System. Anesth Analg. 1999;88:1069-72.

12. Palumbo P, Tellan G, Perotti B, Pacilè MA, Vietri F, Illuminati G. Modified Post Anaesthetic Discharge Scoring System for monitoring outpatients discharge. Ann Ital Chir. 2013;84:661-5.

13. Marshall S, Chung F. Assessment of "home readiness": discharge criteria and postdischarge complications. Curr Opin Anaesthesiol. 1997;10:445-50.

14. Yun H, Ip V, Chung F. Escort accompanying discharge after ambulatory surgery: a necessity or a luxury? Curr Opin Anaesthesiol. 2009;22:748-54.

15. Chung F, Imasogie N, Ho J, Ning $X$, Prabhu A, Curti B. Frequency and implications of ambulatory surgery without a patient escort. Can J Anaesth 2005; 52:1022-6.

16. Apfelbaum JL, Walander CA, Grasela TH, Wise P, McLeskey Ch, Roizen MF, et al. Eliminating Intensive Postoperative Care in Same-day Surgery Patients Using Short-acting Anesthetics. Anesthesiology 2002;97:66-74.

17. Dexter F, Tinker J. Analysis of strategies to decrease postanesthesia care unit costs. Anesthesiology 1995;82:94-101.

18. Williams BA, Kentor ML, Williams JP, Vogt MT, DaPos SV, Harner CD, et al. PACU Bypass after Outpatient Knee Surgery Is Associated with Fewer Unplanned Hospital Admissions but More Phase II Nursing Interventions. Anesthesiology 2002;97:981-8.

19. Awad IT, Chung F. Factors affecting recovery and discharge following ambulatory surgery. Can J Anesth. 2006;53:858-72.

20. Armstrong KA, Semple JL, Coyte PC. Replacing Ambulatory Surgical Follow-Up Visits With Mobile App Home Monitoring: Modeling CostEfective Scenarios. J Med Internet Res. 2014; 16:e213

21. Warner MA, Shields SE, Chute CG. Major morbidity and mortality within 1 month of ambulatory surgery and anesthesia. JAMA 1993;270:1437-41.

22. Chung F, Mezei G. Factors contributing to a prolonged stay after ambulatory surgery. Anesth Analg. 1999;89:1352-9.

23. Junger A, Klasen J, Benson M, Sciuk G. Factors determining length of stay of surgical day-case patients. Eur J Anaesthesiol. 2001;18:314-21.

24. Chung F, Ritchie E, Su J. Postoperative pain in ambulatory surgery. Anesth Analg. 1997;85:808-16. is:

25. Pavlin DJ, Chen C, Penaloza DA, Polissar NL, Buckley FP. Pain as a factor complicating recovery and discharge after ambulatory surgery. Anesth Analg. 2002;95:627-34.

26. Apfel CC, Laara E, Koivuranta M, Greim CA, Roewer N. A simplified risk score for predicting postoperative nausea and vomiting: conclusions from cross-validations between two centers. Anesthesiology 1999;91:693-700.

27. Apfel CC, Kranke P, Eberhart LH, Roos A, Roewer N. Comparison of predictive models for postoperative nausea and vomiting. Br J Anaesth. 2002;88:234-40.

28. Nazar C, Bastidas J, Coloma R, Zamora M, Fuentes R. Prevención y tratamiento de pacientes con naúseas y vómitos posoperatorios. Rev Chil Cir. 2017;69:421-28.

29. Imasogie N, Chung F. Effect of return hospital visits on economics of ambulatory surgery. Curr Opin Anaesthesiol. 2001;14:573-78.

30. Fortier J, Chung F, Su J. Unanticipated admission after ambulatory surgerya prospective study. Can J Anesth. 1998:45:612-9.

31. Twersky R, Fishman D, Homel P. What happens after discharge? Return hospital visits after ambulatory surgery. Anesth Analg. 1997;84:319-24. ist:pi:

32. Mezei G, Chung F. Return hospital visits and hospital readmissions after ambulatory surgery. Ann Surg. 1999;230:721-7. [is:

33. Coley KC, Williams BA, DaPos SV, Chen C, Smith RB. Retrospective evaluation of unanticipated admissions and readmissions after same day surgery and associated costs. J Clin Anesth. 2002; 14:349-53. is is:

34. Fleisher LA, Pasternak LR, Lyles A. A novel index of elevated risk of inpatient hospital admission immediately following outpatient surgery. Arch Surg. 2007;142:263-8. 